International Journal of Engineering \& Technology, $7(2)(2018) 533-535$
International Journal of Engineering \& Technology
SPC
Website: www. sciencepubco.com/index.php/IJET
doi: $10.14419 /$ ijet. 772.10709
Research paper

\title{
A novel review on various energy efficient routing algorithms in wireless sensor networks
}

\author{
Abhilasha Jain ${ }^{1}$ *, Ashok Kumar Goel ${ }^{2}$ \\ ${ }^{1}$ CSE Department, GZS Campus College of Engineering and Technology, \\ Maharaja Ranjeet Singh Punjab Technical University, Punjab, India \\ ${ }^{2}$ ECE Department, GZS Campus College of Engineering and Technology, \\ Maharaja Ranjeet Singh Punjab Technical University, Punjab, India \\ *Corresponding author E-mail: abd_jain@ rediffmail.com,
}

\begin{abstract}
In the past few decades, Wireless sensor networks have exhibited a significant amount of growth and have been used in various applications like traffic control, environment monitoring etc. It comprises an accumulation of sensor nodes that sense the data from their surroundings and relay it to the base station. The network suffers from the limited energy constraints since the sensor nodes are mobile nodes and they run out of battery after a considerable amount of time. To overcome this, a certain level of heterogeneity is introduced among the nodes in terms of energy consumption to sustain the overall network lifetime. Various protocols are developed to prolong the network longevity. Among those, PEGASIS (Power-Efficient Gathering in Sensor Information Systems) and LEACH (Low- Energy Adaptive Clustering Hierarchy) are the significant ones, which ensures power-efficient gathering of the data in the sensor networks. This paper attempts to discuss the different aspects of PEGASIS and LEACH and their advantages and disadvantages in detail.
\end{abstract}

\section{Introduction}

Recent amelioration in the MEMES-based technology and digital electronics has made it possible to design the low -power micro sensors [1 3 [ 3 that are sensitive to assimilate the data from their surroundings. These micro sensors are deployed in an area which can perceive, locate and collect the information and forward it to a centralized authority like base station. These sensors are deployed in huge amounts autonomously and can connect the logical world and the physical world [4] by sending the data to the destination terminal in either a single hop or multi-hop manner. The major flaw of these networks is plenty of sensor nodes and limited energy. Due to various limitations inherent in a wireless network like limited transmission capability, battery power etc., effective routing becomes a strenuous task. With an aim to make communication reliable and efficient, routing protocols $[5,6]$ are employed that aid in discovering and maintaining the paths that are energyefficient. On the basis of the network structure, routing protocols are divided into three categories: flat routing, cluster based or hierarchical routing and location based routing.

In flat routing protocols, each and every node in the network performs the same task and senses the data from the surroundings. No nodes in the system have any special processing capability whatsoever. Such protocols are well suited for small networks; however, they fail miserably when applied to large scale networks. In hierarchical routing protocols, nodes in the network play different roles depending upon their capabilities. The whole network is segmented into clusters and a node in each cluster is designated as cluster head which has special tasks from the regular nodes. It contributes considerably in improving the scalability, energy efficiency and lifetime of the network. In this paper, the authors vividly discuss the two major cluster based routing protocols:
LEACH [7] and PEGASIS [8] and perform their comparative analysis in detail.

Rest of the paper is organized as follows: Section 2 discusses elaborately the related work in the literature, section 3 compares the protocols: LEACH and PEGASIS in terms of various parameters, section 4 discusses issues in the protocols and section 5 concludes the study.

\section{Related work}

Extensive research has been carried out so far to develop efficient routing protocols suited for the vigorous wireless sensor network environment.

Chourse et.al [9] discussed LEACH protocol in detail. Several versions of LEACH like TL-LEACH, M-LEACH, and LEACH-C have been proposed. A mathematical methodology is proposed to calculate the cluster head and various merits and demerits of LEACH are also discussed. The main issue in this is the distance between the cluster head and the base station. As the distance between the cluster head and the base station increases, the protocol is no longer scalable.

$\mathrm{Xu}$ et.al [10] proposed a game-theoretic approach for efficient clustering in wireless sensor networks (GAEC). It aims at choosing the cluster heads based on game theory and slave cluster heads are also chosen in case of failure of the original cluster head. Once a cluster head that is chosen fails, the other nodes in the network are reset which consumes a considerable amount of energy.

J.Yang et.al. [11] developed a new data transmission scheme based on unequal clustering (DTUC). It aims to solve the hot spot problem which occurs due to multihop transmission mode of data transfer. In this scheme, clusters are created considering the distance and probability of the nodes from the sink node. 
Vidhyapriya et. al [12] put forth a multipath adaptive routing technique which is energy efficient and adaptive. It utilizes multiple paths between source and destination. Adaptive routing techniques have an extra benefit over non-adaptive techniques as they reduce the routing overheads and prevent the sensor nodes from running out of their battery prematurely.

An improvised version of ant colony algorithm for chain formation in PEGASIS [13] was proposed by Guo et.al. It aims at forming the chain which makes the paths even-distributed. Linping et.al. [14] employed the concept of double cluster heads for PEGASIS. In this, the whole network is divided into various data levels and the cluster heads are designated as primary cluster heads and secondary cluster heads. The task of the primary cluster heads is to relay the data at a certain level from the chain to the secondary cluster heads. On the other hand, secondary cluster heads are responsible for transmitting the data at the lower level from the primary cluster heads to higher level clusters.

An improved Energy efficient PEGASIS-based protocol (IEEPB) was developed by Sen et.al [15]. It compares the distance between the neighbouring nodes twice and hence prevents the formation of long links between them during the chain formation. The leader node is chosen by considering the normalized value of distance between the base station and nodes and the nodes energy.

\section{Comparison of two cluster based routing protocols: $\mathrm{LEACH}$ and pegasis}

\subsection{LEACH: Low -energy adaptive clustering hierar- chy}

LEACH is one of the state-of-the-art protocols in WSNs developed by Heinzelman et.al [7]. It is an adaptive clustering protocol in which the sensors in the network organizes themselves into clusters and randomly select a cluster head in the cluster. The cluster heads $(\mathrm{CHs})$ are not fixed in this protocol as compared to the other conventional clustering protocols. If they would have been fixed initially, those sensors which have limited battery may be chosen to be the $\mathrm{CH}$ initially would die out soon leading to the cluster failure. In order to avoid draining out the battery of a single sensor, the high energy sensor nodes are being rotated randomly to be the cluster heads. Apart from this, energy consumption is minimized to a large extent in LEACH because it performs data fusion in which amount of data is being compressed while it is forwarded from the cluster heads to the base station. The whole operation consists of two phases: Setup phase and steady phase. In the setup phase, the nodes in the network take the crucial decision of becoming a $\mathrm{CH}$ for the current round which depends upon various factors like percentage of $\mathrm{CHs}$ and the number of times a node has become $\mathrm{CH}$ which depends on the following formula:

$f(x)=\left\{\begin{aligned} \frac{P}{1-P\left(r \bmod \left(\frac{1}{P}\right)\right)}, & \text { if } n \in G \\ & 0, \text { otherwise }\end{aligned}\right.$

Where $\mathrm{P}$ is the percentage of $\mathrm{CHs}, \mathrm{r}$ is the number of rounds and $\mathrm{G}$ is the member nodes that have not been elected as $\mathrm{CH}$ in the past $1 / \mathrm{P}$ rounds. Once a $\mathrm{CH}$ is elected, it sends an advertisement message to all the neighbouring nodes. Based on the received signal strength, the nodes join the nearest cluster. After joining, the nodes send a membership message to the respective cluster head. In order to utilize the maximum energy, the nodes are randomly chosen. In the steady phase, the nodes in the cluster transmit the data to the respective cluster heads and the cluster heads aggregate the data and transmit it to the base station. TDMA/CDMA MAC is used to avoid collisions.

\subsection{Pegasus: power efficient gathering in sensor infor- mation systems}

PEGASIS is an improvement over LEACH developed by Lindsey et.al. In this, a chain is formulated by the nodes in the chain construction phase and a 'leader node' is chosen using greedy algorithm techniques. The node which initiates the data transfer transmits the node to its immediate node in the chain, which appends its own information and forwards it to the next node in the chain. This process is repeated until the data reaches the leader node, which is closest to the base station. The leader node then compresses the data as much as possible to improve the energy efficiency and send it to the base station ultimately. The energy distribution is uniform among the nodes, since all the nodes in the network only have to transmit the data through the chain. Only the leader node is vested with the task of aggregating the data and compressing it. To evenly distribute the energy load among the nodes, the nodes are rotated to become leader nodes time to time. For constructing the chain, each node must have a global knowledge of the whole network. Table 1 shows the merits and demerits of LEACH and PEGASIS. Table 2 shows the comparative study of LEACH and PEGASIS in terms of various parameters of a network.

Table 1: Merits and Demerits of LEACH and PEGASIS

\begin{tabular}{|c|c|c|}
\hline $\begin{array}{l}\text { Routing } \\
\text { Scheme }\end{array}$ & MERITS & DEMERITS \\
\hline LEACH [7] & $\begin{array}{l}\text { - Avoids unnecessary collisions of CHs due to use of } \\
\text { TDMA } \\
\text { - Load sharing between the nodes. } \\
\text { Provides scalability as most of the communication } \\
\text { is confined to clusters. } \\
\text { Excessive energy consumption is avoided due to } \\
\text { allocated time slots. }\end{array}$ & $\begin{array}{l}\text { Lack of uniform distribution and load balancing as CHs are select- } \\
\text { ed on the basis of probability } \\
\text { Not suitable for large scale networks as it uses single hop commu- } \\
\text { nication } \\
\text { - Dynamic clustering leads to extra overheads } \\
\text { - Energy hole and coverage problems }\end{array}$ \\
\hline PEGASIS [8] & $\begin{array}{l}\text { - Uniform distribution of energy load } \\
\text { - } \quad \text { Since clusters are formed dynamically, overheads } \\
\text { are reduced } \\
\text { - } \quad \text { Data transmission is decreased. } \\
\text { - } \quad \text { Rotation of leader nodes. }\end{array}$ & $\begin{array}{l}\text { - } \quad \text { Nodes become bottleneck due to long delays } \\
\text { - } \quad \text { Not very scalable network } \\
\text { - } \quad \text { Not suited for dynamic topologies } \\
\text { - } \quad \text { Requires global knowledge of the network. }\end{array}$ \\
\hline
\end{tabular}

Table 2: Comparative Study of Leach and Pegasis

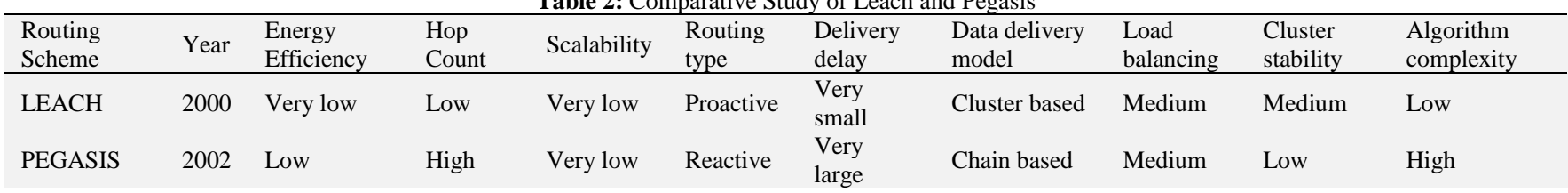




\section{Issues in LEACH and pegasis}

Despite several advantages of cluster based routing protocols, there are various issues that need to be addressed to enhance the efficiency of routing protocols. The issues are discussed below:

1) Selection of Cluster heads (CHs): It plays a vital role in a cluster based wireless sensor network. The $\mathrm{CH}$ so chosen must be a high energy node as it has the crucial tasks of aggregating the data from the nodes in the cluster to the base station. At the same time, in order to balance the energy load in the network, the $\mathrm{CHs}$ have to be rotated at regular intervals based on some scheme. In a multi hop transmission, $\mathrm{CHs}$ have to be changed.

2) Scalability: Both LEACH and PEGASIS suffer miserably from the scalability issues. The clustering techniques proposed in these protocols are not adaptable amidst new deployments of the nodes in an already large networks

3) Energy efficiency: Despite large efforts to improve the energy efficiency in LEACH and PEGASIS, there is still scope to enhance it a little bit more. Experimental results exhibit PEGASIS is better than LEACH in terms of energy efficiency.

4) Heterogeneity of nodes: Since nodes deployed are heterogeneous, it is a challenge to sustain the network lifetime because most crucial nodes like cluster heads in LEACH and Leader nodes in PEGASIS tend to consume more energy than others in spite of load balancing.

\section{Conclusion}

While designing the routing protocols for WSNs, energy efficiency should be kept in mind as these networks are constrained by limited energy. This paper attempts to discuss two most popular cluster based routing protocols available in the literature: LEACH and PEGASIS. The performance of the two protocols is compared based on several parameters like hop count, routing type, energy efficiency etc. The merits and demerits of the protocols are also discussed distinctly. The inference drawn from this comparative study provides a deep insight into the facts that PEGASIS is better than LEACH in terms of energy efficiency and network limited in limited transmission range. On the other hand, in terms of hop count and delivery delay, LEACH performs better. Our future work will be to discuss other cluster based routing protocols.

\section{References}

[1] Chandrakasan, A., Amirtharajah, R., Cho, S., Goodman, J., Konduri, G., Kulik, J. \& Wang, A. (1999). Design considerations for distributed micro sensor systems. In Custom Integrated Circuits, 1999. Proceedings of the IEEE 1999 (pp. 279-286). IEEE https://doi.org/10.1109/CICC.1999.777291.

[2] Clare, L. P., Pottie, G. J., \& Agre, J. R. (1999, July). Selforganizing distributed sensor networks. In Unattended Ground Sensor Technologies and Applications (Vol. 3713, pp. 229-238). International Society for Optics and Photonics. https://doi.org/10.1117/12.357138.

[3] Dong, M. J., Yung, K. G., \& Kaiser, W. J. (1997, August). Low power signal processing architectures for network micro sensors. In Proceedings of the 1997 international symposium on Low power electronics and design (pp. 173-177). ACM https://doi.org/10.1145/263272.263320.

[4] Srbinovski, B., Magno, M., O'Flynn, B., Pakrashi, V., \& Popovici, E. (2015, April). Energy aware adaptive sampling algorithm for energy harvesting wireless sensor networks. In Sensors Applications Symposium (SAS), 2015 IEEE (pp. 1-6). IEEE.

[5] Al-Karaki, J. N., \& Kamal, A. E. (2004). Routing techniques in wireless sensor networks: a survey. IEEE wireless communications, 11(6), 6-28 https://doi.org/10.1109/MWC.2004.1368893.

[6] Sohraby, K., Minoli, D., \& Znati, T. (2007). Wireless sensor networks: technology, protocols, and applications. John Wiley \& Sons. https://doi.org/10.1002/047011276X.
[7] Heinzelman, W. R., Chandrakasan, A., \& Balakrishnan, H. (2000, January). Energy-efficient communication protocol for wireless micro sensor networks. In System sciences, 2000. Proceedings of the 33rd annual Hawaii international conference on (pp. 10-pp). IEEE.

[8] Lindsey, S., \& Raghavendra, C. S. (2002). PEGASIS: Powerefficient gathering in sensor information systems. In Aerospace conference proceedings, 2002. IEEE (Vol. 3, pp. 3-3). IEEE. https://doi.org/10.1109/AERO.2002.1035242.

[9] Chourse, L. K., Chaurse, D., \& Chourse, P. (2012). A Review on Energy Efficient of Clustering-based Routing Protocol in Wireless Sensor Network. International Journal of Computer Applications, 50(16).

[10] Xu, Z., Yin, Y., Wang, J., \& Kim, J. U. (2014). A game-theoretic approach for efficient clustering in wireless sensor networks. International Journal of Hybrid Information Technology, 7(1), 67-80. https://doi.org/10.14257/ijhit.2014.7.1.06.

[11] Yang, J., \& Zhang, D. Y. (2009). A data transmission mechanism for wireless sensor networks using unequal clustering. Journal of Xi'an Jiaotong University, 43(4), 14-17.

[12] Vidhyapriya, R., \& Vanathi, P. T. (2007). Energy efficient adaptive multipath routing for wireless sensor networks. IAENG International Journal of Computer Science, 34(1).

[13] Guo, W., Zhang, W., \& Lu, G. (2010, March). PEGASIS protocol in wireless sensor network based on an improved ant colony algorithm. In Education Technology and Computer Science (ETCS), 2010 Second International Workshop on (Vol. 3, pp. 64-67). IEEE.

[14] Linping, W., Wu, B., Zhen, C., \& Zufeng, W. (2010, August). Improved algorithm of PEGASIS protocol introducing double cluster heads in wireless sensor network. In Computer, Mechatronics, Control and Electronic Engineering (CMCE), 2010 International Conference on (Vol. 1, pp. 148-151). IEEE. https://doi.org/10.1109/CMCE.2010.5609618.

[15] Sen, F., Bing, Q., \& Liangrui, T. (2011, July). An improved energyefficient pegasis-based protocol in wireless sensor networks. In Fuzzy Systems and Knowledge Discovery (FSKD), 2011 Eighth International Conference On (Vol. 4, pp. 2230-2233). IEEE. 